13,06

\title{
Получение, структура и диэлектрические характеристики монокристаллов и тонких пленок $\mathrm{Sr}_{0.61} \mathrm{Ba}_{0.39} \mathrm{Nb}_{2} \mathrm{O}_{6}$
}

\author{
(C) А.В. Павленко ${ }^{1,2}$, Л.И. Ивлева ${ }^{3}$, Д.В. Стрюков ${ }^{1}$, А.П. Ковтун ${ }^{1}$, А.С. Анохин ${ }^{1}$, П.А. Лыков ${ }^{3}$ \\ ${ }^{1}$ Южный научный центр РАН, \\ Ростов-на-Дону, Россия \\ ${ }^{2}$ Южный федеральный университет, \\ Ростов-на-Дону, Россия \\ ${ }^{3}$ Институт общей физики им. А.М. Прохорова РАН, \\ Москва, Россия \\ E-mail: Antvpr@mail.ru
}

(Поступила в Редакцию 18 июля 2018 г.

В окончательной редакции 7 сентября 2018 г.)

Исследованы структура, динамика решетки и диэлектрические характеристики монокристаллов $\mathrm{Sr}_{0.61} \mathrm{Ba}_{0.39} \mathrm{Nb}_{2} \mathrm{O}_{6}$ (SBN-61) и тонких пленок SBN-61/(001)MgO. Из анализа температурно-частотных зависимостей диэлектрической проницаемости и тангенса угла диэлектрических потерь в области $10-500 \mathrm{~K}$ для кристалла SBN-61 определены температурные интервалы фазовых превращений. Установлено, что пленки $\mathrm{SBN}-61 /(001) \mathrm{MgO}$ имеют тетрагональную симметрию, характерную для кристалла SBN-61, но отличные от последнего параметры ячейки.

Работа выполнена в рамках гос. задания ЮНЦ РАН (тема № гос. регистрации 01201354247), при частичной поддержке гранта Volkswagen Foundation Az: 90261 of $29^{\text {th }}$ February 2017, гос. задания Минобрнауки России (проект № 3.6371.2017/8.9) и гранта Президента РФ № МК-4100.2018.2.

DOI: 10.21883/FTT.2019.02.47140.217

\section{1. Введение}

Сегнетоэлектрические (СЭ) твердые растворы (ТР) $\mathrm{Sr}_{x} \mathrm{Ba}_{1-x} \mathrm{Nb}_{2} \mathrm{O}_{6}\left(\mathrm{SBN}_{x}\right)$ в настоящее время являются одними из наиболее перспективных материалов для применения в микроэлектронике, элементах энергонезависимой памяти и нелинейной оптики, и достаточно хорошо изучены в виде порошка, керамики и монокристаллов [1]. С увеличением содержания $\mathrm{Sr}$ в данных ТP температура фазового перехода (ФП) из тетрагональной СЭ фазы в параэлектрическую (ПЭ) снижается и одновременно усиливается релаксорное поведение, которое, как предложено в [1], определяется статистикой заселения катионами $\mathrm{Sr}$ и Ва каналов $A 1$ и $A 2$ в структуре типа тетрагональных вольфрамовых бронз (ТВБ). Это приводит, в том числе, к формированию в них достаточно сложной доменной структуры. В [2] показано, что структуры, образующиеся при переключении из монодоменного состояния в SBN, качественно отличаются от классических доменов и представляет собой квазирегулярные ансамбли изолированных клиновидных нанодоменов с заряженными доменными границами. В [3] отмечено, что при уменьшении соотношения $\mathrm{Sr} / \mathrm{Ba}$ снижается заселенность только пятиугольных структурных каналов и параллельно увеличивается расщепление позиций атомов $\mathrm{Ba}$ и $\mathrm{Sr}$ в этих каналах. В последние годы все большее внимание стало уделяться экспериментальным и теоретическим исследованиям свойств гетероструктур на основе тонких пленок $\mathrm{SBN}_{x}$. Это обусловлено тем, что в таких интенсивно развивающихся направлениях, как микро- и наноэлектроника, микроэлектромеханические системы (МЕМС), СЭ применяются преимущественно в виде тонких пленок [4]. Настоящая работа посвящена изучению структуры, динамики решетки и диэлектрических характеристик (в интервале температур $10-600 \mathrm{~K})$ монокристаллов SBN-61 и тонких пленок SBN-61/(001)MgO.

\section{2. Объекты. Методы получения и исследования}

Монокристаллы $\mathrm{Sr}_{0.61} \mathrm{Ba}_{0.39} \mathrm{Nb}_{2} \mathrm{O}_{6}$ (SBN-61) были выращены модифицированным способом Степанова в Институте общей физики им. А.М. Прохорова РАН. Твердый раствор состава SBN-61 был получен методом твердофазного синтеза при температуре $1473 \mathrm{~K}$ из исходных компонентов $\mathrm{SrCO}_{3}, \mathrm{BaCO}_{3}, \mathrm{Nb}_{2} \mathrm{O}_{5}$ марки „осч“. Керамическая мишень изготавливалась в отделе интеллектуальных материалов и нанотехнологий НИИ физики ЮФУ.

Газоразрядное RF-напыление пленок SBN-61 толщиной $300 \mathrm{~nm}$ при повышенных давлениях чистого кислорода на монокристаллическую подложку (001) $\mathrm{MgO}$ производилось на установке Плазма-50-СЭ [5] (ЦКП НИИ физики ЮФУ). Начальная температура подложки $\sim 673 \mathrm{~K}$, давление кислорода в камере - 0.5 Torr, ВЧ мощность $-110 \mathrm{~W}$.

Структурное совершенство пленок, параметры элементарной ячейки в направлении нормали к плоскости 
подложки, а также ориентационные соотношения между пленкой и подложкой устанавливались рентгенографированием на дифрактометре „ДРОН-4-07““ методом $\theta / 2 \theta$

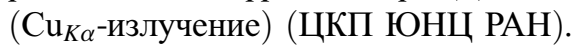

Температурные зависимости относительной диэлектрической проницаемости $\varepsilon^{\prime} / \varepsilon_{0},\left(\varepsilon_{0}\right.$ - диэлектрическая постоянная) и тангенса угла диэлектрических потерь, $\operatorname{tg} \delta$, образцов при $T=20-700 \mathrm{~K}$ и диапазоне частот $f=10^{3}-10^{6} \mathrm{~Hz}$ получали с помощью измерительного стенда на базе LCR-метра Agilent 4980A. Для проведения измерений на грани кристаллов наносилась паста auromal 38 (DODUCO GmBH).

Для получения спектров комбинационного рассеяния света (КРС) в диапазоне $50-1000 \mathrm{~cm}^{-1}$ использовалось поляризованное излучение аргонового лазеpa $(\lambda=514.5 \mathrm{~nm})$ и микро-КРС спектрометр Renishaw inVia c edge-фильтром, позволяющим записывать спектры начиная с $50 \mathrm{~cm}^{-1}$. Спектр КРС регистрировался по схеме обратного рассеяния с помощью оптического микроскопа Leica ( $\times 50$ объектив), диаметр лазерного пучка на образце составлял порядка $2 \mu \mathrm{m}$. Полученные спектры КРС были скорректированы на температурный фактор Бозе-Эйнштейна. Измерения проводились в лаборатории спектроскопии КРС кафедры „Нанотехнология“ Южного федерального университета.

\section{3. Экспериментальные результаты и обсуждение}

На рис. 1, 2 и в таблице приведены результаты исследований диэлектрических характеристик (температурно-частотных зависимостей относительной диэлектрической проницаемости, измеренных вдоль $\left(\varepsilon_{\|}^{\prime} / \varepsilon_{0}\right)$ и перпендикулярно $\left(\varepsilon_{\perp}^{\prime} / \varepsilon_{0}\right)$ полярной оси $\left.c\right)$ монокристалла SBN-61. В интервале температур $T=300-500 \mathrm{~K}$ при понижении температуры наблюдается увеличение $\varepsilon_{\|}^{\prime} / \varepsilon_{0}$ (при $T=360-500 \mathrm{~K}$ дисперсия $\varepsilon_{\|}^{\prime} / \varepsilon_{0}(f)$ практически отсутствует) и формирование максимумов, $T_{m 1}$, сдвигающихся в область более высоких температур по мере увеличения $f$ и связанных с СЭ $\rightarrow$ ПЭ ФП $(\mathrm{P} 4 \mathrm{bm} \rightarrow \mathrm{P} 4 \mathrm{~b} 2) \quad[1,6]$, и „горба“ при $T \sim 335-340 \mathrm{~K}$ (рис. $2, b)$, положение которого не зависит от $f$. Дальнейшее понижение температуры приводит к монотонному снижению $\varepsilon_{\|}^{\prime} / \varepsilon_{0}$ и уменьшению диэлектрической дисперсии (при $T<100 \mathrm{~K}$ в анализируемом диапазоне $f$ дисперсия исчезает - вставка на рис. $1, a)$.

Характеристики монокристалла SBN-61

\begin{tabular}{c|c}
\hline$\varepsilon_{\|}^{\prime} / \varepsilon_{0}(T=300 \mathrm{~K})$ & 1165 \\
$\varepsilon_{\perp}^{\prime} / \varepsilon_{0}(T=300 \mathrm{~K})$ & 447 \\
$\varepsilon_{\|\|}^{\prime} / \varepsilon_{0}\left(T=T_{m}\right)$ & 20228 \\
$\varepsilon_{\perp}^{\prime} / \varepsilon_{0}\left(T=T_{m}\right)$ & 614 \\
$T_{m}(f=1 \mathrm{kHz}), \mathrm{K}$ & 345 \\
$T_{d}, \mathrm{~K}$ & 360 \\
$T_{f}, \mathrm{~K}$ & 335.6
\end{tabular}

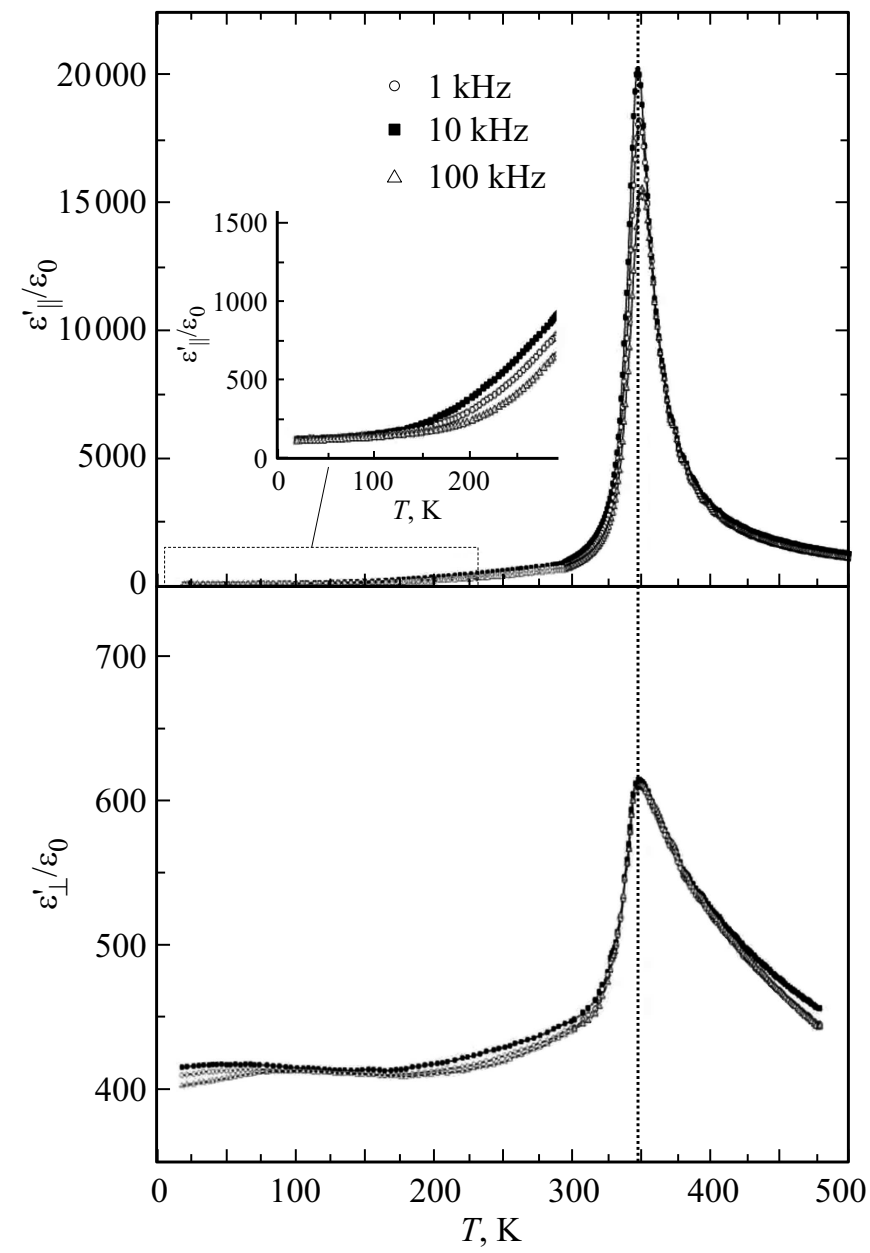

Рис. 1. Зависимости от температуры относительной диэлектрической проницаемости монокристалла SBN-61 на частоте измерительного электрического поля 1,10 и $100 \mathrm{kHz}$ вдоль и перпендикулярно полярной оси $c$.

Из зависимостей $\left(\varepsilon_{\|}^{\prime} / \varepsilon_{0}\right)^{-1}(T)$, иллюстрирующей выполнение закона Кюри-Вейсса, установлено (рис. 2,a), что температура Бёрнса, $T_{\mathrm{d}}$, (температура зарождения полярных нанообластей в СЭ-релаксорах) составляет $360 \mathrm{~K}$. При аппроксимации зависимости $T_{\mathrm{m}}(f)$ наилучшие результаты были достигнуты в случае использования соотношения Фогеля-Фулчера (рис. 2, a, вставка)

$$
f=f_{0} \exp \left(E_{\text {act }} /\left(k\left(T_{\mathrm{m}}-T_{f}\right)\right)\right),
$$

где $f_{0}$ - частота попыток преодоления потенциального барьера $E_{\text {act }}, k-$ постоянная Больцмана, $T_{f}-$ температура Фогеля-Фулчера, интерпретируемая как температура „статического замораживания“ электрических диполей или перехода в состояние дипольного стекла.

Рассчитанные значения $E_{\text {act }}$ и $f_{0}(0.0121 \mathrm{eV}$ и $10^{12} \mathrm{~Hz}$, соответственно) близки к наблюдаемым в СЭ-релаксорах, а величина $T_{f}=335.6 \mathrm{~K}$ позволяет связать выявленные ранее аномалии на кривых $\varepsilon_{\|}^{\prime} / \varepsilon_{0}(T)$ в этой области температур с переходом SBN-61 из релаксорного (нанополярного) состояния в макродоменное, в 
котором, учитывая [7], „обычные“ объемные СЭ домены сосуществуют с приповерхностными наноразмерными доменами.
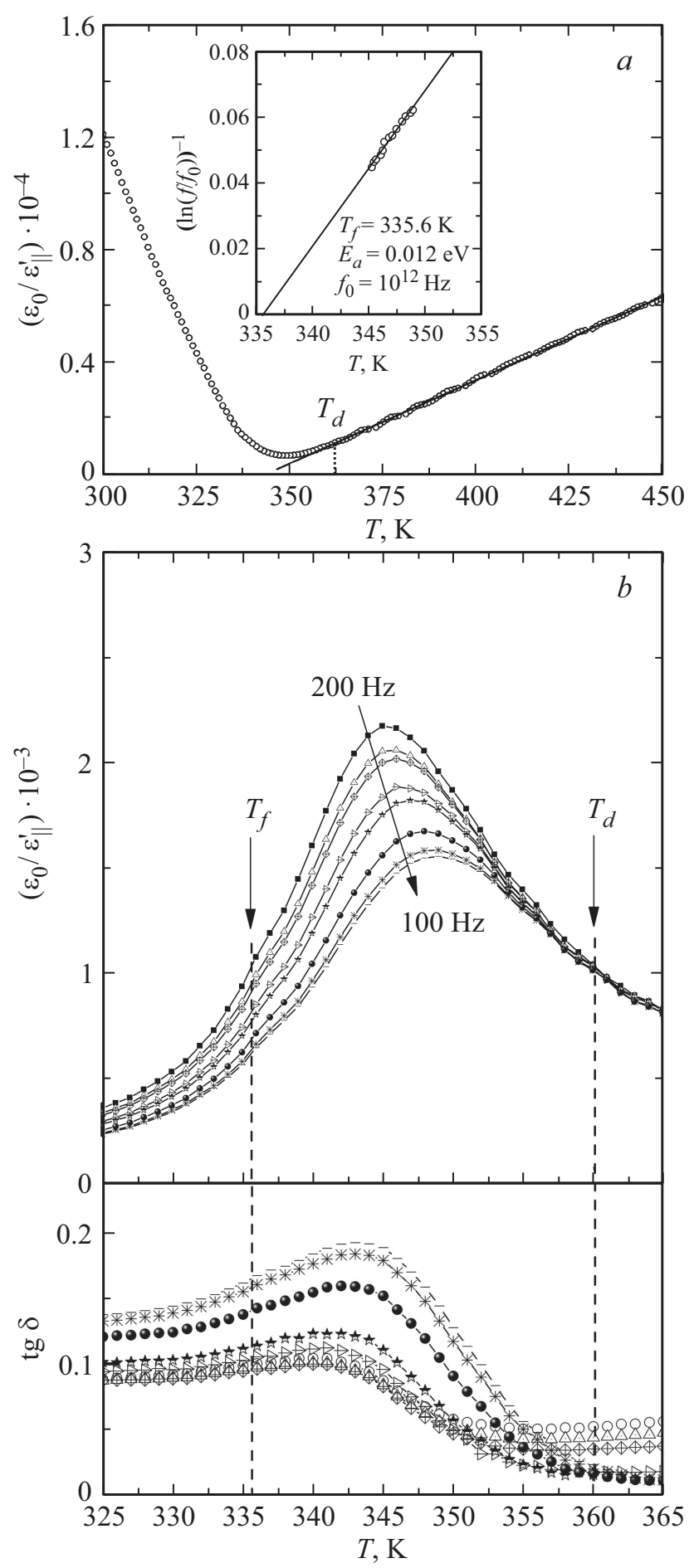

Рис. 2. $а$ - Зависимость $\left(\varepsilon_{\|}^{\prime} / \varepsilon_{0}\right)^{-1}(T)$ при $f=10^{5} \mathrm{~Hz}$ монокристалла SBN-61, прямая линия - иллюстрация выполнения закона Кюри-Вейсса. На вставке - зависимость $\left(\ln \left(f / f_{0}\right)\right)^{-1}(T)$, прямая линия - результат расчета по соотношению Фогеля-Фулчера. $b-$ зависимости $\varepsilon_{\|}^{\prime} / \varepsilon_{0}(T)$ и $\operatorname{tg} \delta(T)$ монокристалла SBN-61 при $f=\left(200 \ldots 10^{5}\right) \mathrm{Hz}$ и $T=(325 \ldots 365) \mathrm{K}$
Как отмечено в $[1,8]$, релаксорные свойства ТР $\mathrm{Sr}_{x} \mathrm{Ba}_{1-x} \mathrm{Nb}_{2} \mathrm{O}_{6}$ обусловлены микроскопическим разупорядочением их структуры. Основу структуры $\mathrm{SBN}_{x}$ составляют два типа кристаллографически независимых $\mathrm{NbO}_{6}$-октаэдров, объединенных кислородными вершинами в трехмерный каркас. Наиболее узкие каналы треугольного сечения (каналы $C$ ) в $\mathrm{SBN}_{x}$ пусты; средние по диаметру каналы четырехугольного сечения $(A 1)$ заполняются только атомами $\mathrm{Sr}$, наиболее крупные каналы пятиугольного сечения $(A 2)$ заполнены атомами $\mathrm{Ba}$ и $\mathrm{Sr}$. По данным [3], заселенность канала $A 1$ атомами $\mathrm{Sr}$ слабо зависит от состава, а релаксорные характеристики $\mathrm{SBN}_{x}$ в основном определяются статистикой заселения каналов $A 2$ атомами $\mathrm{Ba}$ и Sr. Достаточно близкие значения $T_{f}, T_{m 1}$, и $T_{d}$ говорит о достаточно узком температурном размытии $Ф П$ для TP состава SBN-61 в сравнении с другими СЭ-релаксорами.

При рассмотрении кривых $\varepsilon_{\perp}^{\prime} / \varepsilon_{0}(T)$ установлено, что при $T=200-500 \mathrm{~K}$ в объекте наблюдаются, в целом, аналогичные закономерности, однако ярко проявилась свойственная $\mathrm{SBN}_{x}$ сильная анизотропия диэлектрических характеристик в области $П Э \rightarrow$ СЭ ФП (см. таблицу). При $T<200 \mathrm{~K}$, как и в [8], на зависимостях $\varepsilon_{\perp}^{\prime} / \varepsilon_{0}(T)$ формируются размытые частото-зависимые максимумы $\left(T_{m 2}\right)$, сдвигающиеся в область более высоких температур по мере увеличения $f\left(T_{m 2}(f=1 \mathrm{kHz})=53 \mathrm{~K}\right.$, $\left.T_{m 2}(f=100 \mathrm{kHz})=101 \mathrm{~K}\right)$. Согласно результатам работ $[9,10]$, посвященных исследованию акустических параметров, структуры и пироэффекта в $\mathrm{SBN}_{x}$, появление этих аномалий связано с размытым низкотемпературным СЭ $\rightarrow$ СЭ фазовым переходом из тетрагональной в моноклинную фазу. Последнее характеризуется появлением в $\mathrm{SBN}_{x}$ компоненты спонтанной поляризации в перпендикулярном оси $c$ направлении [8], а учитывая результаты [8] ее кластеры могут существовать и давать вклад в диэлектрический отклик и при $T>200 \mathrm{~K}$.

Таким образом, результаты исследования диэлектрических характеристик монокристаллов SBN-61 показали, что в объекте в температурном интервале $10-500 \mathrm{~K}$ происходит достаточно сложная последовательность фазовых превращений. Это необходимо учитывать при прогнозировании характеристик тонких пленок SBN-61, так как возникающие на стадии формирования гетероструктуры деформационные поля могут привести к сдвигу температур ФП в объекте или же появлению новых фазовых состояний, не реализуемых в монокристаллах [4]. Учитывая выше сказанное, были проведены исследования и сопоставлены структура и динамика решетки монокристаллов и тонких пленок SBN-61.

На полученных $\theta-2 \theta$ рентгенограммах гетероструктуры SBN-61/(001) MgO видны только (001) отражения от пленки и подложки, что свидетельствует об отсутствии примесных фаз (рис. 3,a). Доказательство эпитаксиального роста было получено из $\varphi$-сканирования отражений (113) подложки и (221) пленки SBN-61 

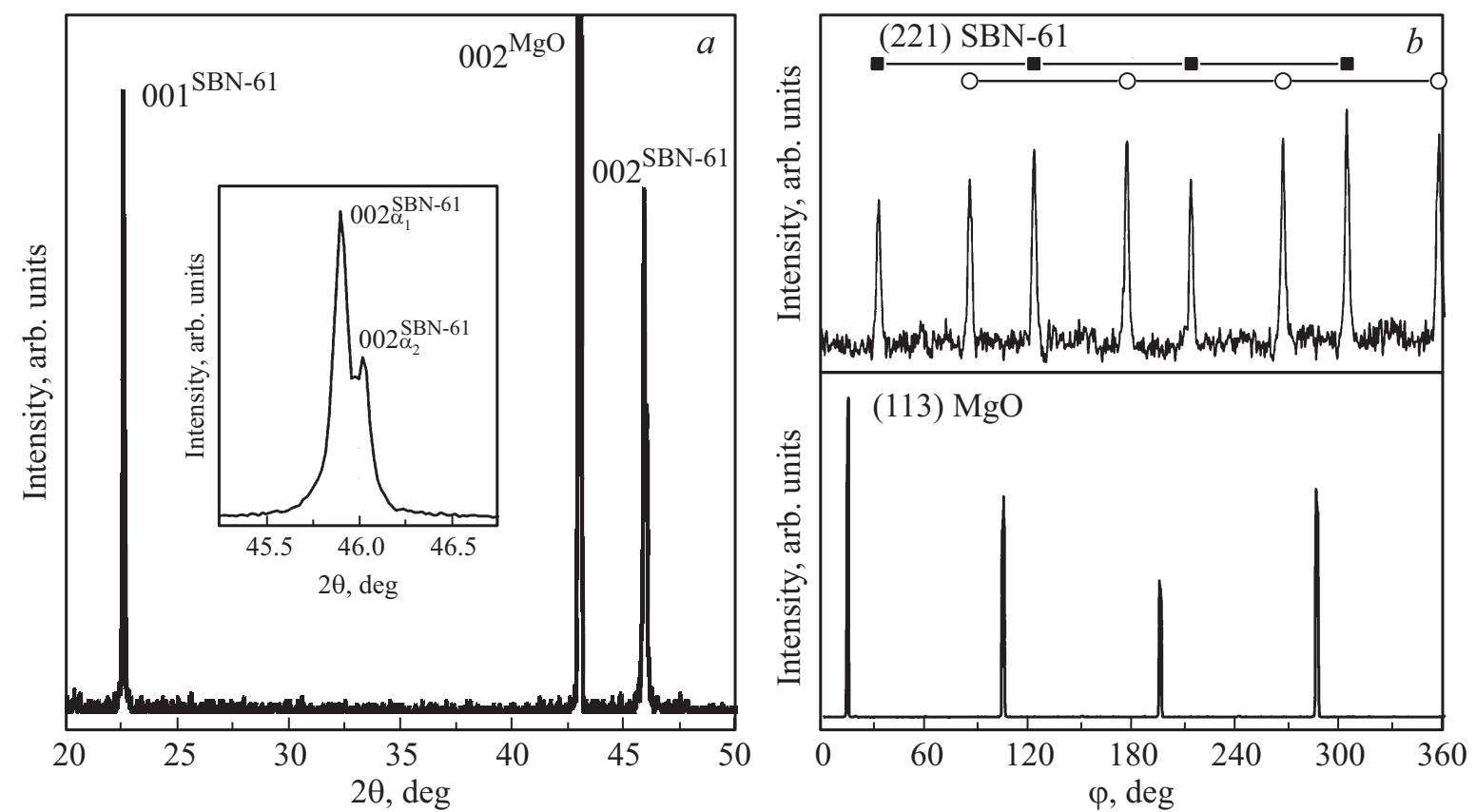

Рис. 3. $a-\theta-2 \theta$ рентгенограмма пленки SBN-61/(001)MgO, на вставке отражение (002) пленки; $b-\varphi$-сканирование отражений (113) подложки MgO и (221) пленки SBN-61, где квадраты и кружки соответствуют ориентационным доменам +18.4 и -18.4 соответственно.

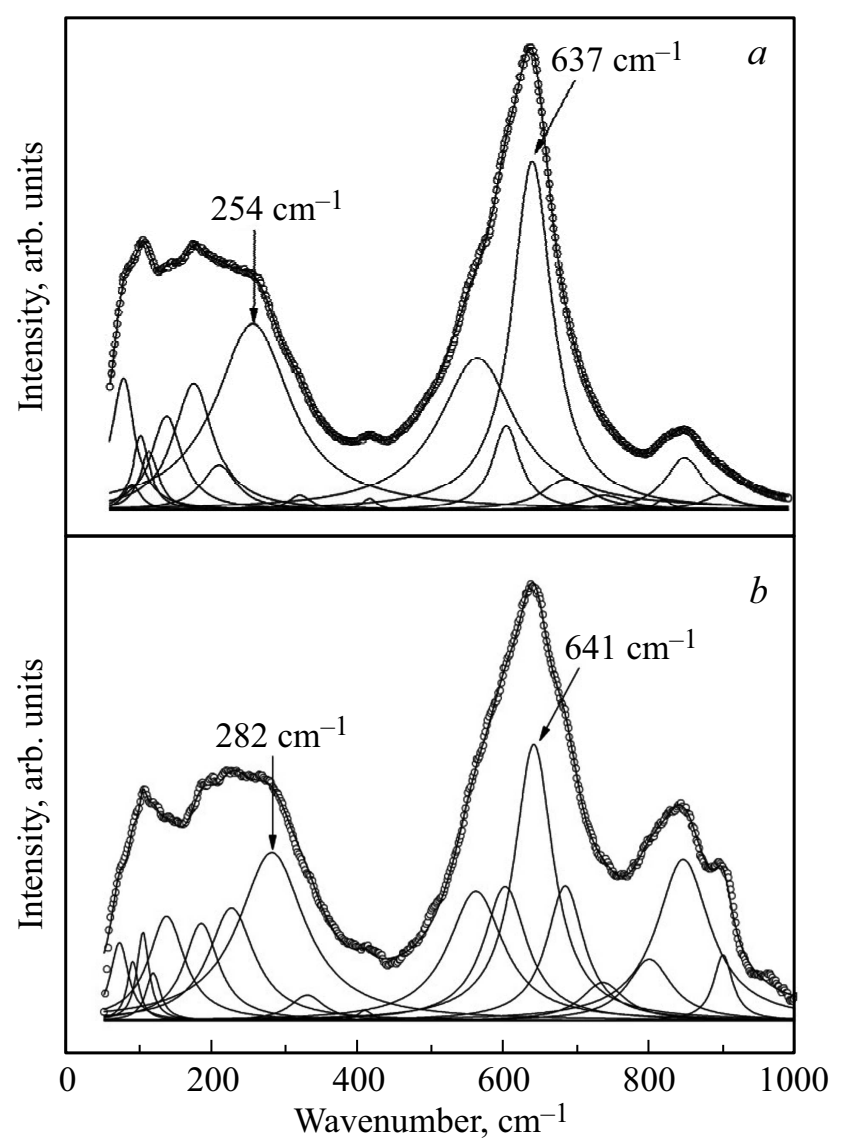

Pис. 4. Спектры КРС кристалла $\mathrm{SBN}-61$ (a) и пленки SBN-61/(001)MgO $(b)$. (рис. $3, b)$. На $\varphi$-сканировании отражения (221) пленки присутствуют восемь отражений что соответствует двум ориентационным доменам в пленке. Кристаллографические оси [001] данных ориентационных доменов повернуты на $\pm 18.4^{\circ}$ относительно оси [001] подложки. Из положений максимумов отражений были определены параметры элементарной ячейки. Следует отметить, что для обоих ориентационных доменов параметры решетки одинаковы, к тому же параметры решетки $a$ и $b$ в плоскости сопряжения равны между собой для обоих ориентационных доменов.

Таким образом, полученные параметры элементарной ячейки пленки $\mathrm{SBN}-61 /(001) \mathrm{MgO}$ равны: $c=$ $=0.3954 \pm 0.0001 \mathrm{~nm} ; a=b=1.245 \pm 0.001 \mathrm{~nm}$. Сравнивая полученные параметры элементарной ячейки с параметрами монокристаллического образца $\left(a_{b}=1.2456 \mathrm{~nm}, c_{b}=0.3936 \mathrm{~nm}\right)$ видно, что пленка практически не имеет деформации в плоскости сопряжения, однако сильно растянута в направлении полярной оси [001] (двумерные деформации элементарной ячейки $\left.\varepsilon_{33}=\left(c-c_{b}\right) / c_{b} \cdot 100 \%=0.45 \%\right)$. Из кривой качания установлено, что вертикальная разориентировка менее $1^{\circ}$, а из $\varphi$-сканирования - азимутальная разориентировка меньше $2.7^{\circ}$, что говорит о достаточно высоком качестве полученной гетероструктуры.

На рис. 4 показаны спектры КРС кристалла SBN-61 и пленки SBN-61/(001)MgO. Для определения параметров отдельных линий экспериментальные спектры КРС были аппроксимированы набором лоренцианов. Согласно правилам отбора для $\mathrm{TP} \mathrm{SBN}_{x}$ существуют 135 колебательных мод $(3 \times 45)$, включая 3 акустические моды. 
Из пяти неприводимых представлений $A 1, A 2, B 1, B 2$ и $E$, только $A 2$ является неактивным и в КРС- и в ИК-спектрах. Моды симметрии $B$ активны в КРС, а моды $A 1$ и $E$ активны и в КРС, и в ИК. Однако, соответствующие линии, наблюдаемые в экспериментальных спектрах кристаллов $\mathrm{SBN}_{x}$ [11] достаточно широки, а их количество значительно меньше теоретически предсказанных мод [12]. Это связывается, в первую очередь, с катионной неупорядоченностью в структуре ТВБ [6], и, как следствие, - с нарушением правил отбора по волновому вектору в центре зоны Бриллюэна [13]. Также для мод с близкими частотами возможно вырождение их в одну полосу, что вносит дополнительный вклад в уширение КРС-спектра. Все это позволяет изучить только ограниченное число спектральных особенностей. Кристалл $\mathrm{MgO}$ не имеет КРС-активных мод первого порядка, однако может наблюдаться спектр второго порядка [14]. Линия $900 \mathrm{~cm}^{-1}$ в спектре SBN-61/(001) MgO относится к спектру второго порядка подложки $\mathrm{MgO}$.

Как видно из рис. 4 , все характерные особенности для спектров структур типа ТВБ сохраняются и в спектрах пленки SBN-61/(001)MgO, что также свидетельствует о сохранении в пленках тетрагональной симметрии. Однако положение частот нескольких линий сдвинуты относительно аналогичных линий в кристалле SBN-61. Наибольший вклад в интенсивность спектра кристалла SBN-61 вносят три широкие полосы на частотах $254 \mathrm{~cm}^{-1}, 637 \mathrm{~cm}^{-1}$ и $846 \mathrm{~cm}^{-1}$. Первые две интенсивные линии, наблюдавшиеся и в ряде других сегнетоэлектрических кристаллов, как показано в [15], являются $A 1$ фононами и относятся к внутренним колебаниям $\mathrm{NbO}_{3}\left(\mathrm{NbO}_{6}\right)$ октаэдра, соответствующие движению ионов вдоль оси $Z$. Линия на частоте $846 \mathrm{~cm}^{-1}$ может быть отнесена к $B 1$ и $B 2$ фононам, которые не дают вклад в поляризацию [13]. В пленке SBN-61/(001) MgO происходит некоторое перераспределение интенсивностей линий и их уширение, наблюдается свдиг первых двух интенсивных полос на частоты $282 \mathrm{~cm}^{-1}$ и $641 \mathrm{~cm}^{-1}$, соотвественно. При этом полоса в районе $846 \mathrm{~cm}^{-1}$ не изменяет своего частотного положения.

Сдвиг и уширение этих линий в спектрах КРС, с нашей точки зрения, могут быть обусловлены деформацией кристаллической решетки и дополнительным структурным разупорядочением в пленке SBN-61.

\section{4. Выводы и заключение}

Исследование диэлектрических характеристик монокристаллов SBN-61 в интервале $10-500 \mathrm{~K}$ позволило установить температурные области существования двух размытых фазовых переходов: низкотемпературного сегнето $\rightarrow$ сегнетоэлектрического фазового перехода при вариациях кристаллической структуры и фазового перехода из сегнетоэлектрической в параэлектрическую фазу $(345-355 \mathrm{~K})$.
При исследовании структуры и динамики решетки гетероструктуры SBN-61/(001)MgO установлено, что элементарной ячейке пленки SBN-61, выращенной в рамках одностадийной технологии роста при повышенных давлениях кислорода, свойственна тетрагональная симметрия. В сравнении с монокристаллическим образцом решетка SBN-61/(001) MgO практически не деформирована в плоскости сопряжения с подложкой, но вытянута в направлении полярной оси. Ранее нами было показано, что в случае $c$-ориентированных пленок $\mathrm{SBN}-50 / \mathrm{Pt}_{2} / \mathrm{Al}_{2} \mathrm{O}_{3}$ более низкие деформационные эффекты приводят к росту $T_{d}$ и снижению величины показателя преломления луча [16]. Это позволяет ожидать в гетероструктурах $\mathrm{SBN}-61 /(001) \mathrm{MgO}$, в частности, усиления оптической анизотропии и смещения фазового перехода СЭ $\rightarrow$ ПЭ в более высокотемпературную область.

Полученные результаты могут быть использованы при получении и исследовании свойств гетероструктур на основе твердых растворов ниобатов бария-стронция.

\section{Список литературы}

[1] Ю.С. Кузьминов. Сегнетоэлектрические кристаллы для управления лазерным излучением. Наука, М. (1982). $400 \mathrm{c}$.

[2] В.Я. Шур, В.А. Шихова, Д.В. Пелегов, А.В. Иевлев, Л.И. Ивлева. ФТТ 53, 2195 (2011).

[3] Т.С. Черная, Б.А. Максимов, Т.Р. Волк, Л.И. Ивлева, В.И. Симонов. ФТТ 42, 1668 (2000).

[4] К.А. Воротилов, В.М. Мухортов, А.С. Сигов. Интегрированные сегнетоэлектрические устройства. Энергоатомиздат, М. (2011). 175 c.

[5] Г.Н. Толмачев, А.П. Ковтун, И.Н. Захарченко, И.М. Алиев, А.В. Павленко, Л.А. Резниченко, И.А. Вербенко. ФТТ 57, 2050 (2015).

[6] P.B. Jamieson. J. Chem. Phys. 48, 5048 (1968).

[7] С.А. Борисов, Н.М. Окунева, С.Б. Вахрушев, А.А. Набережнов, Т.Р. Волк, А.В. Филимонов. ФТТ 55, 295 (2013).

[8] E. Buixaderas, M. Savinov, M. Kempa, S. Veljko, S. Kamba, J. Petzelt, R. Pankrath, S. Kapphan. J. Phys.: Condens. Matter. 17, 653 (2005).

[9] А.С. Пилипенко, А.И. Бурханов, Л.И. Ивлева. ФТТ 51, 543 (2009).

[10] Y. Xu, Z. Li, W. Li, H. Wang, H. Chen. Phys. Rev. B 40, 11902 (1989).

[11] E. Amzallag, T.S. Chang, R.H. Pantell, R.S. Feigelson. J. Appl. Phys. 42, 3254 (1971).

[12] K. Samanta, A.K. Arora, T.R. Ravindran, S. Ganesamoorthy, K. Kitamura, S. Takekawa. Vibrat. Spectroscopy 62, 273 (2012).

[13] R.E. Wilde. J. Raman Spectroscopy 22, 321 (1991).

[14] N.B. Manson, W. Von der Ohe, S.L. Chodos. Phys. Rev. B 3, 1968 (1971).

[15] K.G. Bartlett, L.S. Wall. J. Appl. Phys. 445192 (1973).

[16] А.В. Павленко, А.П. Ковтун, С.П. Зинченко, Д.В. Стрюков. Письма в ЖТФ 44, 30 (2018).

Редактор Т.Н. Василевская 